\title{
MAIN MINERALS AND ORGANIC COMPOUNDS IN COMMERCIAL ROASTED AND GROUND COFFEE: AN EXPLORATORY DATA ANALYSIS
}

\author{
Daneysa Lahis Kalschne ${ }^{\mathrm{a}}$, Nathalia Karen Silva ${ }^{\mathrm{a}}$, Cristiane Canan ${ }^{\mathrm{a}}$, Marta de Toledo Benassi ${ }^{\mathrm{b}}$, Eder Lisandro Moraes \\ Flores $^{\mathrm{c}}$ and Oldair Donizete Leite $\mathrm{c}^{\mathrm{c}, * \text {,(i) }}$ \\ aDepartamento de Alimentos, Universidade Tecnológica Federal do Paraná, 85884-000, Medianeira - PR, Brasil

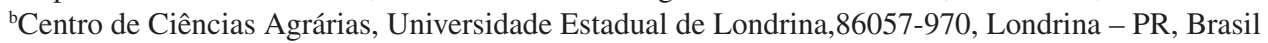 \\ 'Departamento de Química, Universidade Tecnológica Federal do Paraná, 85884-000, Medianeira - PR, Brasil
}

Recebido em 05/08/2020; aceito em 09/09/2020; publicado na web em 15/10/2020

\begin{abstract}
Coffee is one of the most popular beverages in the world, however, little information is found regarding the mineral composition of commercial roasted and ground coffees (RG) and its correlation with organic bioactive compounds. 21 commercial Brazilian RG coffee brands - 9 traditional (T) and 12 extra strong (ES) roasted ones - were analyzed for the $\mathrm{Cu}, \mathrm{Ca}, \mathrm{Mn}, \mathrm{Mg}, \mathrm{K}, \mathrm{Zn}$, and Fe minerals, caffeine, 5-caffeoylquinic acid (5-CQA) and melanoidins contents. For minerals determination by flame atomic absorption spectrometry (FAAS), the samples were decomposed by microwave-assisted wet digestion. Caffeine and 5-CQA were determined by liquid chromatography and melanoidins by molecular absorption spectrometry. The minerals and organic compounds contents association in RG coffee was observed by a principal component analysis. The thermostable compounds (minerals and caffeine) were related to dimension 1 and 2, while 5-CQA and melanoidins were related to dimension 3, allowing for the T coffees segmentation from ES ones.
\end{abstract}

Keywords: caffeine; calcium; filtered coffee; potassium; principal component analysis.

\section{INTRODUCTION}

Brazil is the world's largest coffee producer and exporter, with an estimated production from 57.2 to 62.0 million $60 \mathrm{~kg}$-bags in 2020. ${ }^{1}$ Coffea arabica and Coffea canephora species are the mostly cultivated and marketed ones. According to the Brazilian Coffee Industry Association (ABIC), coffee beverage consumption in Brazil in 2017 was $83 \mathrm{~L}$ per capita and the filtered-brew type prepared with roasted and ground (RG) coffee is the one most consumed. ${ }^{2}$

$\mathrm{RG}$ coffee in Brazil is marketed in 3 categories - gourmet, superior, and traditional/extra-strong - based on the global quality of beverages evaluated by professional cuppers using a 10-grade scale. Taking into account the 896 ABIC-certified brands in 2019, 55\% were graded as traditional/extra-strong, the most sold ones in the Brazilian domestic market. For the traditional/extra-strong category, the raw material may be Coffea arabica or Coffea canephora blends at least NY (New York) 8 types with a maximum 20\% (w/w) of defects and with overall beverage quality graded between 4.5 and $6 .{ }^{3}$ In the Brazilian market, traditional-graded coffees $(\mathrm{T})$ with a more intense roasting process are usually labelled as extra-strong (ES). In short, coffees with more defects are subjected to more aggressive roasting processes to disguise their unpleasant aroma and flavor characteristics.

The mineral content ratio in coffee leaves and beans, mineral supplementation in the plant, and the coffee beverage's quality have been the focus of various studies. ${ }^{4-7}$ Some researches on the mineral content in green coffee are found in order to identify the region of origin. ${ }^{8-12}$ Minerals are stable to the roasting process and thus have an advantage over organic compounds, which were exposed to matter degradation. ${ }^{13}$ However, the mineral content in RG is affected by both environmental and agronomic conditions. During coffee plant nutrition, minerals are usually absorbed from the soil and complemented by fertilizers. ${ }^{12}$

\footnotetext{
*e-mail: oldair.leite@gmail.com
}

In this respect, bioactive organic compounds have been gaining ever increasing attention in the literature for several reasons. Coffee bioactive compounds are well recognized by their health benefits, such as positive memory effects, chronic degenerative diseases prevention, ${ }^{14,15}$ and also an inverse link with the total mortality risk. ${ }^{16}$ Despite their different genetic and environmental conditions from minerals, some bioactive hydrosoluble compounds (e.g., chlorogenic acid and melanoidins) are highly dependent on the roasting process. ${ }^{17,18}$

However, studies on mineral contents in roasted coffees, combined with the bioactive organic compounds determination are scarce in the literature. Some authors have focused solely on the mineral profile ${ }^{19-22}$ and others on organic compounds. ${ }^{23-25}$ The few existing studies combining these two approaches are limited to green coffees beans or coffee brews. ${ }^{8,26}$ No research has been found on inorganic and organic compounds and its relationships in RG coffees - material for most coffee beverages.

In this context, the aim of this study was to provide the main mineral composition ( $\mathrm{Ca}, \mathrm{Cu}, \mathrm{Mn}, \mathrm{Mg}, \mathrm{K}, \mathrm{Zn}$, and Fe contents) and bioactive hydrosoluble compounds (caffeine, 5-caffeoylquinic acid, and melanoidins) on traditional RG coffee types marketed in Brazil.

\section{EXPERIMENTAL}

\section{Reagents, standards, equipment and materials}

Reagents, solvents and analytical grade materials used: ultrapure water obtained with an ultra-purifier system $(18.2 \mathrm{M} \Omega \mathrm{cm}$ resistivity, Master System $^{\circledR}$, Gehaka, São Paulo, Brazil); concentrated nitric acid $\left(\mathrm{HNO}_{3}\right)(65 \% \mathrm{w} / \mathrm{v}$, Sigma Aldrich, St. Louis, USA) - previously purified in a quartz sub-boiling system (model DuoPur ${ }^{\circledR}$, Milestone, Sorisole, Italy); concentrated hydrogen peroxide $\left(\mathrm{H}_{2} \mathrm{O}_{2}\right)(30 \% \mathrm{v} / \mathrm{v}$, Sigma Aldrich, St. Louis, USA); HPLC grade acetonitrile (J.T. Baker, Phillipsburg, USA); acetic acid (purity $\geq 99.8 \%$, Sigma Aldrich, St. Louis, USA); for flame atomic absorption spectrometer (FAAS), the 
aqueous reference solutions containing $\mathrm{Ca}, \mathrm{Cu}, \mathrm{Fe}, \mathrm{K}, \mathrm{Mg}$, $\mathrm{Mn}$, and $\mathrm{Zn}$ prepared from each elemental standard $\left(1000 \mu \mathrm{g} \mathrm{mL} \mathrm{m}^{-1}\right.$, SCP Science, Baie-d'Urfé, Canada) by serial dilutions. The analysis accuracy procedure was verified using a standard reference material (Embrapa RM-Agro E1001 (FO-01/12), Brachiaria brizantha cv Marandu) digested under similar conditions applied for coffee samples. For HPLC analysis, the following chromatographic standards were used: caffeine and 5-caffeoylquinic acid (5-CQA) (Sigma Aldrich, St. Louis, USA) and an Acclaim ${ }^{\circledR}$ TM Polar advantage II C18 column $(3 \mu \mathrm{m}, 120$ A, 4.6 x 150 mm) (Thermo Fisher Scientific, Germering, Germany).

\section{Coffee samples}

21 RG coffee commercial samples were purchased from a local supermarket in the municipality of Medianeira, Paraná, Brazil. All samples weighted $500 \mathrm{~g}$ and were vacuum-packed. 9 of them were labelled as traditional (T) and 12 as extra strong (ES). The RG coffees color-characterization was done by lightness $\left(\mathrm{L}^{*}\right)^{27}$ measurements in triplicate using a colorimeter (CR-400, Konica Minolta, Tokyo, Japan). The analysis was carried out under an integrating sphere and a $45^{\circ}$ viewing angle (lighting $\mathrm{d} / 45$ and illuminating $\mathrm{D}$ ).

\section{Microwave digestion and mineral determinations}

Microwave digestion was performed according to Amorim Filho et al. in triplicate. ${ }^{19}$ Each sample was weighed $(0.400 \mathrm{~g})$ in $50 \mathrm{~mL}$ PTFE-TFM closed vessels (standing $310^{\circ} \mathrm{C}$ and 40 bar), added with $3 \mathrm{~mL} \mathrm{HNO}_{3}(65 \% \mathrm{w} / \mathrm{v})$ and $2 \mathrm{~mL} \mathrm{H}_{2} \mathrm{O}_{2}(30 \% \mathrm{v} / \mathrm{v})$ and left open at room temperature for $20 \mathrm{~min}$. The samples were subjected to wet digestion using a microwave oven (Multiwave $\mathrm{GO}^{\circledR}$, Anton Paar, Graz, Austria). The USEPA 3052 digestion method was used by linearly increasing the temperature to $180{ }^{\circ} \mathrm{C}$ for $4.5 \mathrm{~min}$, kept for 9.5 min and then cooled.

Mineral determination was carried out using a flame atomic absorption spectrometer (FAAS) equipped with a Deuterium background correction system (model AA 240-FS, Varian, Australia). K was determined by emission mode, whereas all other analytes were determined by absorption mode. The Schinkel modifier $(0.5 \% \mathrm{v} / \mathrm{v})$ was added to all digested samples and reference solutions. External calibration with a 7-point analytical curve for each analyte was performed at the concentration range described in Table 1. The other FAAS operational conditions for each analyte are shown in Table 1.

\section{Organic compounds extraction and caffeine, 5-CQA, and melanoidins determination}

Simultaneous caffeine and 5-CQA analyses and melanoidins estimates were performed as per Kalschne et al.. ${ }^{28}$ Aqueous extracts were obtained in triplicate for the organic compounds determination.
Each coffee sample $(0.5000 \mathrm{~g})$ was extracted with $30 \mathrm{~mL}$ ultrapure water at $80{ }^{\circ} \mathrm{C}$ for $10 \mathrm{~min}$ and filtered using qualitative paper.

For HPLC analysis, a $1 \mathrm{~mL}$ extract aliquot was diluted in $5.0 \mathrm{~mL}$ acetic acid: water $(5: 95 \mathrm{v} / \mathrm{v})$. The extract was filtered $(0.45 \mu \mathrm{m})$ and injected $(20 \mu \mathrm{L})$ into the chromatograph (Ultimate 3000, Thermo Scientific, Germering, Germany). The mobile phase comprised of acetic acid/ultrapure water (5:95 v/v) (A) and acetonitrile (B), and the following gradient elution was used: $1 \mathrm{~min}, 5 \% \mathrm{~B} ; 5 \mathrm{~min}, 13 \%$ $\mathrm{B}$ with $0.6 \mathrm{~mL} \mathrm{~min}^{-1}$. Caffeine and 5-CQA detection were set at 272 and $320 \mathrm{~nm}$, respectively, and identification was based on retention times and UV spectra. Quantification was performed in triplicate $\left(\mathrm{R}^{2} \geq 0.999\right.$ and $\left.\mathrm{P}<0.001\right)$ by external standardization using 6-point analytical curves (20 to $60 \mu \mathrm{g} \mathrm{mL}^{-1}$ for caffeine and 0.5 to $30 \mu \mathrm{g} \mathrm{mL} \mathrm{m}^{-1}$ for 5-CQA).

Melanoidins were estimated by diluting $0.6 \mathrm{~mL}$ of aqueous extract in $1.4 \mathrm{~mL}$ ultrapure water to achieve a $5 \mathrm{mg}$ coffee $\mathrm{mL}^{-1}$ concentration. Absorbance was measured at $420 \mathrm{~nm}$ using a spectrophotometer (Perkin Elmer, Lambda XLS, Beaconsfield, UK). The melanoidins content was estimated based on the $1.1289 \mathrm{~L} \mathrm{~g}^{-1} \mathrm{~cm}^{-1}$ absorptivity value proposed by Tagliazucchi et al.. ${ }^{29}$ A coffee brew was used as melanoidins source to obtain an analytical curve (7 points in triplicate, $\mathrm{R}^{2}=1$ ) at a 1 to $7 \mathrm{mg}$ coffee $\mathrm{mL}^{-1}$ concentration range corresponding to a 0.092 to 1.001 absorbance range. The analysis was performed in triplicate and the results expressed in $\mathrm{mg}$ of melanoidins $100 \mathrm{~g}^{-1}$ of coffee.

\section{Data analysis}

The data obtained were expressed as average \pm standard deviation, with the averages compared by the Tukey test $(p \leq 0.05)$. Moreover, for the mineral and organic profile overview, all data were analyzed by Principal Component Analysis (PCA) and Person's correlation $(\mathrm{p} \leq 0.05)$ using the Statistica 8.0 software.

\section{RESULTS AND DISCUSSION}

The lowest average $\mathrm{L}^{*}$ value for ES coffees $(19.70 \pm 1.25)$ suggests that they were subjected to a more intense roasting process compared to T coffees $(20.75 \pm 1.42)$. However, Souza et al. (2010) observed no difference on the average $\mathrm{L}^{*}$ value for T (19.5) and ES (20.5) Brazilian RG coffees, respectively. Such results reinforced that the ES coffee does not necessarily present a darker color.

When analyzing samples from different Brazilian brands, origins, and processing techniques, it was observed that the extra strong coffees had a slightly lower $\mathrm{L}^{*}$ value than the traditional ones.

\section{Coffee samples mineral contents}

The commercial RG coffee presented a mineral content ranging from 18832 to $28707 \mu \mathrm{g} \mathrm{g}^{-1}$ for $\mathrm{K}$; 2025 to $2779 \mu \mathrm{g} \mathrm{g}^{-1}$ for $\mathrm{Mg}$; 881 to

Table 1. FAAS instrumental conditions for $\mathrm{Cu}, \mathrm{Ca}, \mathrm{Mn}, \mathrm{Mg}, \mathrm{K}, \mathrm{Zn}$, and Fe determination in coffees

\begin{tabular}{|c|c|c|c|c|c|c|c|}
\hline FFAS conditions & $\mathrm{Cu}$ & $\mathrm{Ca}$ & $\mathrm{Mn}$ & $\mathrm{Mg}$ & $\mathrm{K}$ & $\mathrm{Zn}$ & $\mathrm{Fe}$ \\
\hline Wavelength (nm) & 327.4 & 232.0 & 403.1 & 285.2 & 766.5 & 213.9 & 248.3 \\
\hline Spectral band width (nm) & 0.5 & 0.5 & 0.5 & 0.5 & 0.1 & 0.5 & 0.5 \\
\hline Hollow cathode lamp current (mA) & 4 & 4 & 5 & 4 & - & 5 & 5 \\
\hline Air flow $\left(\mathrm{L} \mathrm{min}{ }^{-1}\right)$ & 10 & 10 & 10 & 10 & 10 & 10 & 10 \\
\hline Acetylene $\left(\mathrm{L} \min ^{-1}\right)$ & 2.2 & 2.2 & 2.2 & 2.2 & 2.2 & 2.2 & 2.2 \\
\hline Sample aspiration rate $\left(\mathrm{mL} \min ^{-1}\right)$ & 4.0 & 4.0 & 4.0 & 4.0 & 4.0 & 4.0 & 4.0 \\
\hline Linear range $\left(\mu \mathrm{g} \mathrm{mL}^{-1}\right)$ & $0.25-2.0$ & $0.25-2.0$ & $0.25-2.0$ & $0.25-2.0$ & $0.25-2.0$ & $0.25-2.0$ & $0.25-2.0$ \\
\hline
\end{tabular}


$1861 \mu \mathrm{g} \mathrm{g}^{-1}$ for $\mathrm{Ca} ; 76.2$ to $194.5 \mu \mathrm{g} \mathrm{g}^{-1}$ for $\mathrm{Fe} ; 20.08$ to $41.50 \mu \mathrm{g} \mathrm{g}^{-1}$ for $\mathrm{Mn} ; 14.37$ to $24.39 \mu \mathrm{g} \mathrm{g}^{-1}$ for $\mathrm{Cu}$, and 4.68 to $10.58 \mu \mathrm{g} \mathrm{g}^{-1}$ for $\mathrm{Zn}$ (Table 2).

The mineral content was in the range found in the literature for RG coffees (commercial Coffea arabica, Coffea canephora, blends of both species or non-identified coffee of different origins and roasting degrees). Unlike various organic compounds, the minerals found in coffee are not affected by the roasting process $;^{30}$ and they are nearly entirely found in the final beverage due to their water solubility. ${ }^{31}$ Different researches have described macronutrient contents ranging from 11370 to $29039 \mu \mathrm{g} \mathrm{g}^{-1}$ for $\mathrm{K}, 476$ to $2840 \mu \mathrm{g} \mathrm{g}^{-1}$ for $\mathrm{Mg}$, and 513 to $2235 \mu \mathrm{g} \mathrm{g}^{-1}$ for Ca (Table 3). The Fe micronutrient content ranged from 12 to $282.3 \mu \mathrm{g} \mathrm{g}^{-1}$, from 12.1 to $44.66 \mu \mathrm{g} \mathrm{g}^{-1}$ for $\mathrm{Mn}$, from 8.8 to $30.1 \mu \mathrm{g} \mathrm{g}^{-1}$ for $\mathrm{Cu}$, and from 3.2 to $36.9 \mu \mathrm{g} \mathrm{g}^{-1}$ for $\mathrm{Zn} .{ }^{19-22,32-33}$ Further mineral content details found in other studies are seen in Table 3.

For the macronutrient content, $\mathrm{K}$ was the main mineral present on coffee followed by $\mathrm{Mg}$ and $\mathrm{Ca}$, similar to that previously described by other researchers. ${ }^{19-22}$ According to Martinez et al., the K requirement for the coffee plant is close to that of $\mathrm{N}$, with the highest amount of mineral found in coffee beans. ${ }^{4} \mathrm{Fe}$ was the fourth most abundant mineral found in coffee samples, followed by $\mathrm{Mn}, \mathrm{Cu}$ and $\mathrm{Zn}$ as previously described by other authors (Table 3). ${ }^{19-22}$ Moreover, Debastiani et al. reported that $\mathrm{K}$ and $\mathrm{Mg}$ are extracted by the drip brewing process, while $\mathrm{Ca}, \mathrm{Fe}, \mathrm{Cu}$ and $\mathrm{Zn}$ may be absorbed by spent coffee, which has a sponge-like behavior. ${ }^{32}$

\section{Caffeine, 5-CQA and melanoidins content in coffee samples}

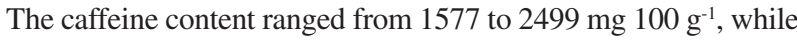

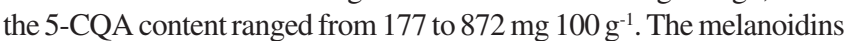

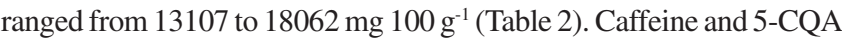
content in commercial roasted and ground coffees fell within the range found in the literature for Coffea arabica, Coffea canephora, blends and commercial coffees of different origins and various roasting degrees. The content ranges reported by other studies were from 1070

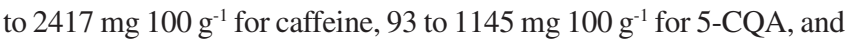

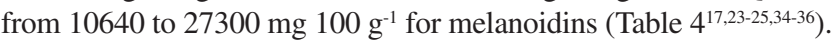

Commercial coffees in Brazil do not usually show the material (Arabica or blended species) or roasting degree on their information labels, but some assumptions may be made based on the composition. For thermostable caffeine, it is well known that Coffea canephora has higher contents than Coffea arabica ones. ${ }^{31,36}$ For 5-CQA, in addition to species variations (Coffea canephora have lower 5-CQA content than Coffea arabica), which is less significant, the chlorogenic acids undergo many changes during the roasting process such as isomerization, epimerization, lactonization, and degradation to lowmolecular-weight compounds during the roasting process, ${ }^{37}$ which justify their lower content in roasted coffees..$^{17,18}$ Melanoidins are brown bioactive compounds produced during the roasting process by the Maillard reaction. No differences in melanoidins content were observed in the RG coffee species, however, a roasting degree increase usually increases the melanoidins content. ${ }^{17,28,38}$

Table 2. Content of mineral and organic compounds of commercial roasted coffee samples (dry basis)

\begin{tabular}{|c|c|c|c|c|c|c|c|c|c|c|}
\hline Sample & $\begin{array}{c}\mathrm{Cu} \\
\left(\mu \mathrm{g} \mathrm{g}^{-1}\right)\end{array}$ & $\begin{array}{c}\mathrm{Ca} \\
\left(\mu \mathrm{g} \mathrm{g}^{-1}\right)\end{array}$ & $\begin{array}{c}M n \\
\left(\mu g^{-1}\right)\end{array}$ & $\begin{array}{c}\mathrm{Mg} \\
\left(\mu \mathrm{g} \mathrm{g}^{-1}\right)\end{array}$ & $\begin{array}{c}\mathrm{K} \\
\left(\mu \mathrm{g} \mathrm{g}^{-1}\right)\end{array}$ & $\begin{array}{c}\mathrm{Zn} \\
\left(\mu \mathrm{g} \mathrm{g}^{-1}\right)\end{array}$ & $\begin{array}{c}\mathrm{Fe} \\
\left(\mu \mathrm{g} \mathrm{g}^{-1}\right)\end{array}$ & 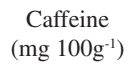 & 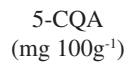 & $\begin{array}{l}\text { Melanoidins } \\
\left(\mathrm{mg}_{\left.100 \mathrm{~g}^{-1}\right)}\right.\end{array}$ \\
\hline $1 \mathrm{~T}$ & $17.41^{\mathrm{d}} \pm 0.45$ & $940^{\mathrm{gh}} \pm 22$ & $24.84^{\mathrm{gh}} \pm 0.30$ & $2025^{\mathrm{ef}} \pm 76$ & $27253^{a} \pm 1213$ & $9.49^{b} \pm 0.18$ & $82.9^{\mathrm{gh}} \pm 2.3$ & $2499^{\mathrm{a}} \pm 12$ & $683^{\mathrm{bc}} \pm 12$ & $15221^{\text {efgh }} \pm 214$ \\
\hline $2 \mathrm{~T}$ & $24.39^{\mathrm{a}} \pm 0.27$ & $1013^{\text {defgh }} \pm 74$ & $27.62^{\operatorname{defg}_{ \pm}} \pm 0.33$ & $2097^{\mathrm{bef}} \pm 148$ & $28707^{\mathrm{abc}} \pm 1502$ & $10.58^{\mathrm{a}} \pm 0.18$ & $120.4^{\mathrm{c}} \pm 13.6$ & $2090^{\mathrm{d}} \pm 25$ & $582^{\mathrm{e}} \pm 14$ & $16733^{\mathrm{bc}} \pm 459$ \\
\hline $3 \mathrm{~T}$ & $18.25^{\mathrm{d}} \pm 0.10$ & $967^{\mathrm{efgh}} \pm 36$ & $27.94^{\text {defg }_{ \pm}} \pm 1.31$ & $2042^{\mathrm{f}} \pm 42$ & $22370^{\mathrm{abcd}} \pm 1473$ & $9.69^{\mathrm{b}} \pm 0.07$ & $116.1^{\mathrm{cd}} \pm 8.1$ & $2116^{\mathrm{d}} \pm 30$ & $699^{b} \pm 23$ & $15407^{\text {efgh }} \pm 82$ \\
\hline $4 \mathrm{~T}$ & $21.94^{b} \pm 0.45$ & $961^{\mathrm{fgh}} \pm 32$ & $33.21^{\mathrm{b}} \pm 0.29$ & $2217^{\text {bcdef }} \pm 117$ & $21721^{\mathrm{bcd}} \pm 629$ & $9.49^{b} \pm 0.32$ & $119.1^{\mathrm{cd}} \pm 2.2$ & $1688^{\mathrm{hi}} \pm 50$ & $712^{\mathrm{b}} \pm 17$ & $13645^{\mathrm{i}} \pm 143$ \\
\hline $5 \mathrm{~T}$ & $14.66^{\mathrm{e}} \pm 0.24$ & $1156^{\mathrm{d}} \pm 51$ & $32.61^{\mathrm{bc}} \pm 0.83$ & $2486^{\text {abcd }} \pm 26$ & $22647^{\mathrm{abcd}} \pm 612$ & $9.31^{\mathrm{bc}} \pm 0.27$ & $194.5^{\mathrm{a}} \pm 4.2$ & $1684^{\mathrm{hi}} \pm 20$ & $872^{a} \pm 21$ & $14908^{\mathrm{gh}} \pm 49$ \\
\hline $6 \mathrm{~T}$ & $14.37^{\mathrm{e}} \pm 1.04$ & $1123^{\mathrm{d}} \pm 29$ & $29.80^{\mathrm{cd}} \pm 0.87$ & $2450^{\mathrm{acd}} \pm 154$ & $22302^{\text {bcd }} \pm 979$ & $10.01^{\mathrm{ab}} \pm 0.14$ & $94.0^{\text {efgh }} \pm 2.3$ & $1896^{\mathrm{f}} \pm 23$ & $497^{\mathrm{f}} \pm 9$ & $16072^{\text {bcde }} \pm 125$ \\
\hline $7 \mathrm{~T}$ & $18.36^{\mathrm{d}} \pm 0.67$ & $904^{\mathrm{h}} \pm 28$ & $30.02^{\mathrm{bcd}} \pm 1.10$ & $2203^{\text {bcdef }} \pm 60$ & $22434^{\mathrm{bcd}} \pm 481$ & $7.99^{\operatorname{defg} g} \pm 0.17$ & $119.3^{\mathrm{c}} \pm 0.5$ & $1793^{\mathrm{g}} \pm 19$ & $642^{\mathrm{cd}} \pm 22$ & $15094^{\mathrm{fgh}} \pm 255$ \\
\hline $8 \mathrm{~T}$ & $17.81^{\mathrm{d}} \pm 0.58$ & $881^{\mathrm{h}} \pm 13$ & $28.19^{\text {def }} \pm 0.22$ & $2334^{\text {bcdef }} \pm 27$ & $21235^{\mathrm{cd}} \pm 689$ & $8.16^{\mathrm{def}} \pm 0.15$ & $111.0^{\mathrm{cde}} \pm 3.3$ & $1916^{\mathrm{f}} \pm 11$ & $450^{g} \pm 7$ & $16863^{b} \pm 161$ \\
\hline $9 \mathrm{~T}$ & $14.49^{\mathrm{e}} \pm 1.29$ & $1107^{\mathrm{def}} \pm 53$ & $23.47^{\mathrm{h}} \pm 0.69$ & $2391^{\text {bcdef }} \pm 169$ & $20574^{\mathrm{d}} \pm 715$ & $8.14^{\mathrm{def}} \pm 0.18$ & $99.1^{\mathrm{defg}_{ \pm}} \pm 2.5$ & $2193^{c} \pm 8$ & $583^{\mathrm{e}} \pm 12$ & $15895^{\text {cdef }} \pm 369$ \\
\hline Mean T & 17.96 & 1006 & 28.63 & 23249 & 23249 & 9.21 & 117.4 & 1986 & 636 & 15538 \\
\hline RSD T & $19 \%$ & $10 \%$ & $11 \%$ & $12 \%$ & $12 \%$ & $10 \%$ & $27 \%$ & $13 \%$ & $20 \%$ & $6 \%$ \\
\hline $1 \mathrm{ES}$ & $18.77^{\mathrm{cd}} \pm 0.90$ & $1074^{\text {defg }} \pm 5$ & $32.47^{\mathrm{bc}} \pm 0.80$ & $2779^{a} \pm 169$ & $23085^{\mathrm{abcd}} \pm 1150$ & $8.63^{\mathrm{cd}} \pm 0.26$ & $112.1^{\mathrm{cde}} \pm 1.1$ & $1789^{\mathrm{g}} \pm 14$ & $412^{\mathrm{gh}} \pm 2$ & $17829^{\mathrm{a}} \pm 258$ \\
\hline $2 \mathrm{ES}$ & $15.26^{\mathrm{e}} \pm 0.51$ & $1341^{\mathrm{c}} \pm 40$ & $28.90^{\mathrm{de}} \pm 2.17$ & $2111^{\text {bcdef }} \pm 145$ & $21579^{\mathrm{bcd}} \pm 323$ & $7.80^{\text {efgh }} \pm 0.50$ & $110.8^{\mathrm{cde}} \pm 9.0$ & $1790^{g} \pm 11$ & $422^{\mathrm{gh}} \pm 14$ & $16842^{\mathrm{b}} \pm 458$ \\
\hline $3 \mathrm{ES}$ & $19.21^{\mathrm{cd}} \pm 0.82$ & $1494^{b} \pm 58$ & $41.50^{\mathrm{a}} \pm 1.13$ & $2404^{\text {bcde }} \pm 158$ & $22565^{\mathrm{abcd}} \pm 589$ & $8.02^{\operatorname{defg}} \pm 0.16$ & $93.1^{\mathrm{efgh}} \pm 3.7$ & $1577^{\mathrm{j}} \pm 11$ & $316^{\mathrm{jk}} \pm 2$ & $14663^{\mathrm{h}} \pm 158$ \\
\hline $4 \mathrm{ES}$ & $15.16^{\mathrm{e}} \pm 0.99$ & $1329^{c} \pm 53$ & $24.78^{\mathrm{gh}} \pm 1.70$ & $2238^{\text {bcdef }} \pm 129$ & $21328^{\mathrm{cd}} \pm 184$ & $7.44^{\mathrm{fgh}} \pm 0.04$ & $76.2^{\mathrm{h}} \pm 1.2$ & $2002^{\mathrm{e}} \pm 14$ & $623^{\mathrm{de}} \pm 13$ & $15900^{\text {cdef }} \pm 361$ \\
\hline $5 \mathrm{ES}$ & $17.61^{\mathrm{d}} \pm 0.33$ & $1408^{\mathrm{bc}} \pm 52$ & $28.27^{\mathrm{def}} \pm 2.11$ & $2479^{\mathrm{ac}} \pm 114$ & $22566^{\mathrm{abcd}} \pm 265$ & $7.75^{\mathrm{efgh}} \pm 0.04$ & $76.4^{\mathrm{h}} \pm 2.8$ & $1726^{\mathrm{gh}} \pm 26$ & $639^{\mathrm{d}} \pm 18$ & $13228^{i} \pm 89$ \\
\hline $6 \mathrm{ES}$ & $24.09^{\mathrm{a}} \pm 1.07$ & $1861^{\mathrm{a}} \pm 81$ & $30.21^{\mathrm{bcd}} \pm 0.77$ & $2495^{\mathrm{ac}} \pm 67$ & $26635^{\mathrm{ab}} \pm 1269$ & $9.46^{b} \pm 0.35$ & $173.3^{\mathrm{b}} \pm 5.5$ & $2160^{\mathrm{cd}} \pm 16$ & $388^{\mathrm{hi}} \pm 6$ & $18062^{\mathrm{a}} \pm 219$ \\
\hline $7 \mathrm{ES}$ & $20.65^{\mathrm{bc}} \pm 0.58$ & $967^{\mathrm{efgh}} \pm 36$ & $25.43^{\mathrm{fgh}} \pm 1.14$ & $2327^{\text {bcdef }} \pm 94$ & $22633^{\mathrm{cd}} \pm 587$ & $8.21^{\mathrm{de}} \pm 0.27$ & $87.7^{\mathrm{fgh}} \pm 3.9$ & $1897^{\mathrm{f}} \pm 24$ & $177^{\mathrm{m}} \pm 4$ & $15745^{\text {defg }_{ \pm}} 520$ \\
\hline $8 \mathrm{ES}$ & $19.34^{\mathrm{cd}} \pm 0.20$ & $1024^{\text {defgh }} \pm 48$ & $24.07^{\mathrm{h}} \pm 0.64$ & $2212^{\text {bcdef }} \pm 92$ & $18832^{\mathrm{d}} \pm 277$ & $7.31^{\mathrm{ghi}} \pm 0.23$ & $104.9^{\mathrm{cdef}} \pm 5.7$ & $2388^{b} \pm 20$ & $520^{\mathrm{f}} \pm 17$ & $16479^{\mathrm{bcd}} \pm 382$ \\
\hline $9 \mathrm{ES}$ & $17.38^{\mathrm{d}} \pm 0.59$ & $961^{\mathrm{fgh}} \pm 20$ & $20.08^{\mathrm{i}} \pm 0.59$ & $2179^{\text {bcdef }} \pm 5$ & $19167^{\mathrm{d}} \pm 127$ & $7.58^{\mathrm{efgh}} \pm 0.27$ & $83.2^{\mathrm{gh}} \pm 4.0$ & $2468^{\mathrm{a}} \pm 26$ & $282^{\mathrm{kl}} \pm 5$ & $17837^{\mathrm{a}} \pm 355$ \\
\hline $10 \mathrm{ES}$ & $17.87^{\mathrm{d}} \pm 0.84$ & $1114^{\mathrm{de}} \pm 76$ & $22.42^{\mathrm{hi}} \pm 1.20$ & $2218^{\text {bcdef }} \pm 103$ & $21707^{\mathrm{cd}} \pm 1038$ & $7.41^{\mathrm{ghi}} \pm 0.22$ & $107.0^{\text {cdef }} \pm 1.3$ & $1957^{\mathrm{ef}} \pm 26$ & $420^{\mathrm{gh}} \pm 14$ & $15670^{\mathrm{efg}} \pm 146$ \\
\hline $11 \mathrm{ES}$ & $18.26^{\mathrm{d}} \pm 0.33$ & $1104^{\mathrm{def}} \pm 42$ & $25.61^{\mathrm{efgh}} \pm 0.24$ & $2106^{\text {bdef }} \pm 55$ & $19846^{\mathrm{d}} \pm 1005$ & $7.02^{\mathrm{hi}} \pm 0.32$ & $113.7^{\mathrm{cde}} \pm 2.7$ & $2111^{\mathrm{d}} \pm 8$ & $341^{\mathrm{ij}} \pm 22$ & $16311^{\mathrm{bcd}} \pm 107$ \\
\hline $12 \mathrm{ES}$ & $18.74^{\text {cd }} \pm 0.17$ & $1080^{\operatorname{defg}_{ \pm}} 57$ & $28.44^{\mathrm{def}} \pm 0.95$ & $2273^{\text {bcdef }} \pm 70$ & $20860^{\mathrm{d}} \pm 423$ & $6.77^{\mathrm{i}} \pm 0.12$ & $100.7^{\mathrm{efg}_{ \pm}}+5.6$ & $1631^{\mathrm{ij}} \pm 46$ & $264^{1} \pm 4$ & $13107^{\mathrm{i}} \pm 280$ \\
\hline Mean ES & 18.53 & 1230 & 27.68 & 21734 & 21734 & 7.78 & 103.3 & 1958 & 399 & 15973 \\
\hline RSD ES & $13 \%$ & $22 \%$ & $11 \%$ & $10 \%$ & $10 \%$ & $9 \%$ & $25 \%$ & $14 \%$ & $35 \%$ & $10 \%$ \\
\hline
\end{tabular}

T: traditional Brazilian coffees; ES: extra strong Brazilian coffees; RSD: relative standard deviation; results expressed by mean \pm standard deviation; different superscript letters in the column indicate differences by the Tukey test $(\mathrm{p} \leq 0.05)$. 
Table 3. Comparative mineral content of roasted coffee samples from different studies

\begin{tabular}{|c|c|c|c|c|c|c|c|c|c|c|}
\hline Reference & Samples & $\begin{array}{l}\text { Digestion } \\
\text { method }\end{array}$ & $\begin{array}{l}\text { Determination } \\
\text { technique }\end{array}$ & $\begin{array}{c}\mathrm{Cu} \\
\left(\mu \mathrm{g} \mathrm{g}^{-1}\right)\end{array}$ & $\begin{array}{c}\mathrm{Ca} \\
\left(\mu \mathrm{g} \mathrm{g}^{-1}\right)\end{array}$ & $\begin{array}{c}\mathrm{Mn} \\
\left(\mu \mathrm{g} \mathrm{g}^{-1}\right)\end{array}$ & $\begin{array}{c}\mathrm{Mg} \\
\left(\mu \mathrm{g} \mathrm{g}^{-1}\right)\end{array}$ & $\begin{array}{c}\mathrm{K} \\
\left(\mu \mathrm{g} \mathrm{g}^{-1}\right)\end{array}$ & $\begin{array}{c}\mathrm{Zn} \\
\left(\mu \mathrm{g} \mathrm{g}^{-1}\right)\end{array}$ & $\begin{array}{c}\mathrm{Fe} \\
\left(\mu \mathrm{g} \mathrm{g}^{-1}\right)\end{array}$ \\
\hline $\begin{array}{l}\text { Current } \\
\text { article }\end{array}$ & & Microwave & FAAS & $14.4-24.4$ & 881-1861 & $20.1-41.5$ & $2025-2779$ & $18832-28707$ & $6.8-10.6$ & $76.2-194.5$ \\
\hline Mean & $\begin{array}{l}\text { zilian commercial ro } \\
\text { coffees }(\mathrm{n}=21)\end{array}$ & & & 18.3 & 1134 & 28.1 & 2289 & 22383 & 8.3 & 109.5 \\
\hline RSD & & & & $15 \%$ & $21 \%$ & $16 \%$ & $8 \%$ & $11 \%$ & $16 \%$ & $26 \%$ \\
\hline 21 & $\begin{array}{l}\text { Coffea arabica roasted coffee from } \\
\text { Brazil, Nicaragua, Honduras, El } \\
\text { Salvador, Colombia and Guatemala } \\
\qquad(\mathrm{n}=9)\end{array}$ & $\begin{array}{l}\text { Digestion } \\
\text { Block }\end{array}$ & ICP-OES & $11.2-17.8$ & 880-1090 & $13.2-44.6$ & $1730-1940$ & $12730-14930$ & $5.06-36.9$ & $40.3-59.8$ \\
\hline 21 & $\begin{array}{l}\text { Coffea canephora roasted coffee } \\
\text { from Ivory Coast, Vietnam, } \\
\text { Cameroon and Uganda }(n=9)\end{array}$ & $\begin{array}{l}\text { Digestion } \\
\text { Block }\end{array}$ & ICP-OES & $13.1-17.2$ & 870-1350 & $12.1-19.8$ & $1460-1820$ & $13890-15410$ & $4.94-31.8$ & $50.6-73.4$ \\
\hline 20 & $\begin{array}{l}\text { Commercial roasted coffees from } \\
\text { Costa Rica, Colombia, Guatemala, } \\
\text { Panama, Ethiopia, Kenya, Sulawesi } \\
\text { and Sumatra }(\mathrm{n}=160)\end{array}$ & $\begin{array}{l}\text { Digestion } \\
\text { Block }\end{array}$ & ICP-OES & $12.5-18.1$ & 934-1234 & 19-39 & 2058-2410 & $17500-19600$ & $6.51-8.03$ & $12-31$ \\
\hline 22 & Commercial roasted coffee $(\mathrm{n}=75)$ & $\begin{array}{l}\text { Dry muffle } \\
\text { incineration }\end{array}$ & FAAS & $12.1-20.1$ & $513-1620$ & $16.5-40.6$ & 800-2840 & $11750-15850$ & $3.2-16.2$ & $20.3-67.8$ \\
\hline 22 & $\begin{array}{c}\text { Commercial Coffea } \\
\text { arabica coffee from India, } \\
\text { Australia, Tanzania, Peru, Cuba, } \\
\text { Timor, Zambia, Honduras and } \\
\text { Indonesia }(\mathrm{n}=60)\end{array}$ & $\begin{array}{l}\text { Dry muffle } \\
\text { incineration }\end{array}$ & FAAS & $8.8-30.1$ & 674-1490 & $17.6-16.6$ & 746-929 & $11370-22750$ & $5.7-14.0$ & $18.4-53.8$ \\
\hline 19 & $\begin{array}{l}\text { Brazilian commercial roasted } \\
\text { coffees }(n=5)\end{array}$ & Microwave & FAAS & $12.5-18.7$ & 1121-1943 & $22.0-36.0$ & 1913-2356 & $19633-26675$ & $4.65-7.14$ & $61.0-282.3$ \\
\hline 19 & $\begin{array}{l}\text { Brazilian commercial roasted } \\
\text { coffees }(n=5)\end{array}$ & $\begin{array}{l}\text { Digestion } \\
\text { Block } \\
\end{array}$ & FAAS & $13.3-18.8$ & $1200-1944$ & $19.8-31.7$ & $1835-2283$ & 20474-27930 & $4.80-15.48$ & 51.4-192.0 \\
\hline 32 & $\begin{array}{l}\text { Brazilian commercial roasted } \\
\text { coffees }(\mathrm{n}=8)\end{array}$ & - & $\mathrm{X}$-rays & $13.0-25.8$ & $1080-2235$ & $26.0-37.0$ & $1758-2596$ & 18142-29039 & 7.1-11.2 & $52-85$ \\
\hline 33 & $\begin{array}{l}\text { Organic coffee from Colombia, } \\
\text { Honduras, Mexico, and Ethiopia } \\
\qquad(\mathrm{n}=4)\end{array}$ & - & $\mathrm{X}$-rays & $10-11$ & $1205-1205$ & $16-32$ & - & $16543-17577$ & $4-6$ & $25-35$ \\
\hline
\end{tabular}

RSD: relative standard deviation.; ICP-OES: inductively coupled plasma optical emission spectrometry; FAAS: flame atomic absorption spectrometry.

Table 4. Comparative caffeine and 5-CQA content and estimative of melanoidins of roasted coffee samples from different studies

\begin{tabular}{|c|c|c|c|c|c|}
\hline Reference & Samples & $\begin{array}{l}\text { Determination } \\
\text { technique }\end{array}$ & 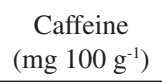 & $\begin{array}{c}\text { 5-CQA } \\
\left(\mathrm{mg} 100 \mathrm{~g}^{-1}\right)\end{array}$ & 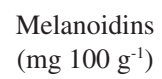 \\
\hline Current article & & HPLC & $1633-2499$ & $264-872$ & $13107-18062$ \\
\hline Mean & Brazilian commercial coffees $(n=21)$ & & 1877 & 478 & 15786 \\
\hline RSD & & & $13 \%$ & $35 \%$ & $9 \%$ \\
\hline 34 & Commercial coffee blends in a sealed package and capsule $(n=2)$ & HPLC & $2280-2417$ & $378-439$ & - \\
\hline 35 & Coffea arabica coffee in different roasting degree $(\mathrm{n}=6)$ & HPLC & $1096-1518$ & - & - \\
\hline 35 & Coffea canephora coffee in different roasting degree $(n=6)$ & HPLC & $1925-2212$ & - & - \\
\hline 25 & Brazilian traditional and extra strong commercial coffees $(n=10)$ & HPLC & - & $114-596$ & - \\
\hline 23 & Brazilian traditional and extra strong commercial coffees $(n=13)$ & HPLC & $1070-1790$ & $140-690$ & - \\
\hline 24 & Coffee arabica roasted at 170 or $200^{\circ} \mathrm{C} / 15 \min (\mathrm{n}=6)$ & HPLC & - & $93-761$ & - \\
\hline 24 & Coffea canephora roasted at 170 or $200^{\circ} \mathrm{C} / 15 \mathrm{~min}(\mathrm{n}=6)$ & HPLC & - & $175-1145$ & - \\
\hline 36 & Coffea arabica and Coffea canephora or blends $(\mathrm{n}=4)$ & Spectrophotometer & - & - & $10640-14590$ \\
\hline 17 & Coffea arabica coffee in different roasting degree $(n=12)$ & Dialysis & - & - & $14400-23600$ \\
\hline 17 & Coffea arabica coffee in different roasting degree $(\mathrm{n}=12)$ & Dialysis & - & - & $18500-27300$ \\
\hline
\end{tabular}

RSD: relative standard deviation.

\section{Principal component analysis}

A three-dimensional consensual solution explained $65 \%$ roasted coffee samples variance (Figure 1). Dimension 1 justified $28 \%$ of variance and it was highly and positively correlated to all mineral contents and, to a lesser extent, to 5-CQA. A positive correlation was observed between some minerals, however, it was weak $(<0.68)$ (data not shown).

Dimension 2 explained $21 \%$ of variance and was mainly positively correlated to caffeine, $\mathrm{K}$, and melanoidins contents. 

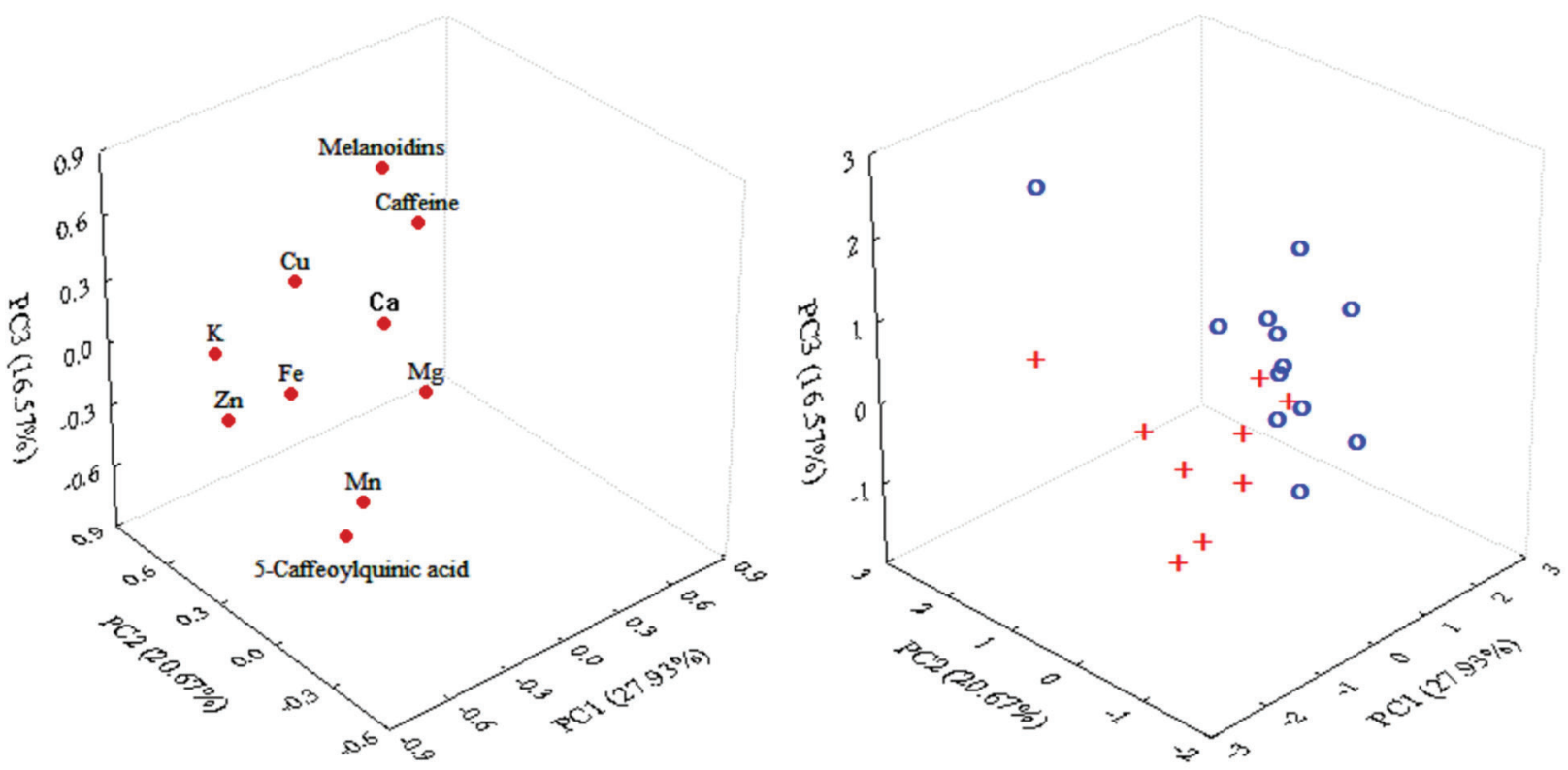

Figure 1. PC1 versus PC2 versus PC3 loadings and scores plots of coffee samples (+: traditional Brazilian coffees; o: extra strong Brazilian coffees)

According to the literature, caffeine could be found in a complex with polyphenols even in green coffee beans. A well-known feature for the two compound classes is their interaction to form an easily found complex: crystalline caffeine potassium chlorogenate $1: 1 .^{37,39}$ This approach may justify the link between caffeine and K content in coffee samples. Moreover, Clemente et al. previously reported the correlation between higher $\mathrm{K}$ content and caffeine content increase on coffee beans. ${ }^{6}$

A negative association between caffeine and melanoidins content may be related to the caffeine thermostability and the melanoidins formation during the roasting process. As reported by Vignoli et al., other bioactive compounds responsible for the coffee's antioxidant activity, such as 5-CQA - except caffeine and melanoidins - have decreased with the roasting process. ${ }^{17}$

To a lesser extent, Dimension 2 correlated negatively caffeine with $\mathrm{Mn}$ and $\mathrm{Mg}$. Caffeine is a secondary metabolite of coffee plant with effects on central nervous system stimulation. Its precursor is theobromine, with three $\mathrm{N}$-methyltransferases involved in its biosynthesis. ${ }^{4}$ According to Suzuki and Takahashi, methyltransferases do not require divalent cations such as $\mathrm{Mg}^{2+}$ and $\mathrm{Mn}^{2+}$. ${ }^{40}$ Since the mineral chelating agents have only partially inhibited both theobromine and caffeine formation, it appears reasonable that the caffeine content increases while the $\mathrm{Mn}$ and $\mathrm{Mg}$ contents decrease on coffee beans. It is a well-known fact that caffeine content is coffee-species-dependant. $\mathrm{T}(1986 \pm 265 ; \mathrm{RSD} 13 \%)$ and ES coffees (1958 $\pm 282 ; \mathrm{RSD} 14 \%)$ caffeine contents were similar considering the average $(\mathrm{P}<0.05)$ and variation (considering RSD). The literature reports a caffeine content

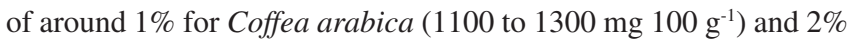
for Coffea canephora (2400 to $2500 \mathrm{mg} 100 \mathrm{~g}^{-1}$ ). ${ }^{15,31}$ In this context, considering that all RG coffee samples evaluated had high caffeine amounts (around 2\%), it is suggested that all samples could have a considerable Coffea canephora amount in their blend.

Dimension 3 explained $16.57 \%$ of variance and was positively correlated to 5-CQA content and negatively correlated to the melanoidins content. Such dimension was correlated to a roasting degree, segmenting $\mathrm{T}$ coffees (at bottom of the plot) from ES ones (top of the plot). Significant beans composition changes occurred during the roasting process due to pyrolysis, caramelization, and Maillard reactions. ${ }^{37}$ Melanoidins were formed by the interaction among polysaccharides, galactomannans, and arabinogalactans (the latter being mostly covalently linked to proteins in green coffee beans), proteins (amino acids), and chlorogenic acids. Therefore, when chlorogenic acids were degraded during roasting, melanoidins were formed (with chlorogenic acids incorporated in their structure). ${ }^{17,31,41}$ The 5-CQA content for T $(636 \pm 126$; RSD 20\%) and ES coffees $(400 \pm 140$; RSD $35 \%)$ suggest that ES coffees were more intensely roasted and had a greater roasting process variation.

The principal component analysis by dimension 3 allowed the coffee segmentation into traditional and extra strong. Dimensions 1 and 2 showed the occurrence of a correlation between minerals and organic compounds (i.e. caffeine), mainly associated with agricultural treatments and agronomic conditions. Since the minerals and caffeine contents had no variation in the roasting process, its proportions would remain in roasted coffee, making them attractive for segmenting commercial coffees with beans that have been produced at various roasting degrees. On the other hand, dimension 3 was closely related to the roasting process, so it separated into more and less roasted coffees, regardless of their origin.

Since both mineral and organic compounds are hydrosoluble, the correlations between them in roasted beans are reflected in the coffee brew.

\section{CONCLUSIONS}

The principal component analysis allows for traditional coffee segmentation from extra strong ones, taking into account the mineral and organic compounds evaluated. The thermostable compounds (minerals and caffeine) are more attractive for roasted and ground coffee correlations.

\section{ACKNOWLEDGMENTS}

This study was financed in part by the Conselho Nacional de Desenvolvimento Científico e Tecnológico (CNPq), Coordenação de Aperfeiçoamento de Pessoal de Nível Superior - Brasil (CAPES) - Finance Code 001, and Fundação Araucária.

\section{REFERENCES}

1. https://www.conab.gov.br/info-agro/safras, accessed September 2020 
2. http://abic.com.br/estatisticas/indicadores-da-industria/indicadores-daindustria-de-cafe-2017/, acessed September 2020.

3. http://abic.com.br/src/uploads/2017/07/2.8.1-Norma-de-qualidade-PQC. pdf, accessed September 2020.

4. Martinez, H. E. P.; Clemente, J. M.; Lacerda, J. S.; Neves, Y. P.; Pedrosa, A. W.; Rev. Ceres 2014, 61, 838.

5. Martinez, H. E. P.; Lacerda, J. S.; Clemente, J. M.; Silva Filho, J. B.; Pedrosa, A. W.; Santos, R. H. S.; Cecon, P. R.; Pesqui. Agropecu. Bras. 2018, 53, 443 .

6. Clemente, J. M.; Martinez, H. E. P.; Alves, L. C.; Finger, F. L.; Cecon, P. R.; Acta Sci. Agron 2015, 37, 297.

7. Lacerda, J. S.; Doctorate Thesis, Universidade Federal de Viçosa, Brazil, 2014.

8. Jeszka-Skowron, M.; Stanisz, E.; Peña, M. P.; LWT - Food Sci. Technol 2016, 73, 243.

9. Mohammed, F.; Guillaume, D.; Dowman, S.; Abdulwali, N.; Microchem. $J$ 2019, 145, 173.

10. Morgano, M. A.; Pauluci, L. F.; Mantovani, M. D.; Mory, E. E. M.; Ciênc. Tecnol. Aliment. 2002, 22, 19.

11. Şemen, S.; Mercan, S.; Yayla, M.; Açıkkol, M.; Food Chem. 2017, 215 , 92.

12. Cruz, R.; Morais, S.; Casal, S; In Processing and Impact on Active Components in Food; Preedy, V. R., ed., Academic Press: Cambridge, 2014, chapter 66.

13. Bitter, N. Q.; Fernandez, D. P.; Driscoll, A. W.; Howa, J. D.; Ehleringer, J. R.; Food Chem 2020, 126602.

14. Hu, G. L.; Wang, X.; Zhang, L.; Qiu, M. H.; Food Funct 2019, 10, 3113.

15. Wang, X.; Lim, L.-T. In Coffee in Health and Disease Prevention; Preedy, V. R., ed.; Elsevier: Amsterdam, 2015, chapter 27.

16. Ding, M.; Satija, A.; Bhupathiraju, S. N.; Hu, Y.; Sun, Q.; Han, J.; Lopez-Garcia, E.; Willett, W.; van Dam, R. M.; Hu, F. B.; Circulation 2015, 132, 2305.

17. Vignoli, J. A.; Viegas, M. C.; Bassoli, D. G.; Benassi, M. T.; Food Res. Int 2014, 61, 279.

18. Dias, R.; Benassi, M.; Beverages 2015, 1, 127.

19. Amorim Filho, V. R.; Polito, W. L.; Gomes Neto, J. A.; Braz. Chem. Soc 2007, 18, 47.

20. Anderson, K. A.; Smith, B. W.; J. Agric. Food Chem 2002, 50, 2068.

21. Martín, M. J.; Pablos, F.; González, A. G.; Food Chem 1999, 66, 365.
22. Grembecka, M.; Malinowska, E.; Szefer, P.; Sci. Total Environ. 2007, $383,59$.

23. Souza, R. M. N.; Canuto, G. A.; Dias, R. C. E.; Benassi, M. T.; Quim. Nova 2010, 33, 885

24. Perrone, D.; Farah, A.; Donangelo, C. M.; Paulis, T.; Martin, P. R.; Food Chem 2008, 106, 859 .

25. Monteiro, M. C.; Trugo, L.; Quim. Nova 2005, 28, 637.

26. Stelmach, E.; Pohl, P.; Szymczycha-Madeja, A.; Food Chem 2015, 182, 302.

27. Bicho, N. C.; Leitão, A. E.; Ramalho, J. C.; Lindon, F. C.; Ciênc. Tecnol. Aliment. 2012, 32, 436.

28. Kalschne, D. L.; Viegas, M. C.; Conti, A. J.; Corso, M. P.; Benassi, M. T.; LWT - Food Sci. Technol2019, 99, 364.

29. Tagliazucchi, D.; Verzelloni, E.; Conte, A.; J. Agric. Food Chem 2010, $58,2513$.

30. Bonnländer, B.; Eggers, R.; Engelhardt, U. H.; Maier, H. In Espresso coffee the science of quality; Illy, A; Viani, R., eds.; Elsevier: Amsterdam, 2019, chapter 4.

31. Rosa, J. S.; Freitas-Silva, O.; Godoy, R. L. O.; Rezende, C. M. In Food Processing Technologies; Jaiswal, A. K., ed., CRC Press: Boca Raton, 2016, chapter 4

32. Debastiani, R.; Santos, C. E. I.; Ramos, M. M.; Souza, V. S.; Amaral, L.; Yoneama, M. L.; Dias, J. F.; Food Res. Int 2019, 119, 297.

33. Cloete, K. J.; Šmit, Z.; Minnis-Ndimba, R.; Vavpetič, P.; Plessis, A.; Roux, S. G.; Pelicon, P.; Food Chem. X 2019, 2, 100032.

34. Ribeiro, V. S.; Leitão, A. E.; Ramalho, J. C.; Lidon, F. C.; Food Res. Int 2014, 61, 39.

35. Casal, S.; Oliveira, M. B.; Ferreira, M. A.; Food Chem 2000, 68, 481.

36. Kalschne, D. L.; Biasuz, T.; Conti, A. J.; Viegas, M. C.; Corso, M. P.; Benassi, M. T.; Food Res. Int 2019, 124, 234.

37. Stefanello, N.; Spanevello, R. M.; Passamonti, S.; Porciúncula, L.; Bonan, C. D.; Olabiyi, A. A.; Rocha, J. B. T.; Assmann, C. E.; Morsch, V. M.; Schetinger, M. R. C.; Food Chem. Toxicol 2019, 123, 298.

38. Almeida, M. B.; Benassi, M. T.; Semina: Ciências Agrárias 2011, 32, 1893.

39. Waldhauser, S. S. M.; Baumann, T. W.; Phytochemistry 1996,42, 985.

40. Suzuki, T.; Takahashi, E.; Biochem. J 1975, 146, 87.

41. Perrone, D.; Farah, A.; Donangelo, C. M.; J. Agric. Food Chem 2012, $60,4265$. 